\title{
Improving discharge from hospital onto home parenteral nutrition
}

\author{
F. Al-Sulttan, M. Baber, M. Bak-Jensen, K. Docherty, A. Karr, H. Middleton, L. Soo and A. Forbes \\ University College London Hospitals NHS Foundation Trust, 235 Euston Road, London NWI 2BU, UK
}

Initiating home parenteral nutrition (HPN) is always a challenge for the patient involved. While it may represent an opportunity to recapture quality of life in chronic progressive intestinal failure (such as from scleroderma or idiopathic intestinal pseudo-obstruction), its need is more often the consequence of an acute event, such as mesenteric infarction or complications of surgery, for which the patient was unprepared and for whom a need for long-term and potentially lifelong intravenous nutrition (IVN) is formidable. It is therefore to be expected that establishment on IVN and the discharge process will be a measured one, with considerable effort and time spent on its planning. However, the mean intervals of $>2$ months before the patient is discharged, reported from leading UK centres, seem excessive on clinical as well as financial grounds, especially given North American expectations of discharge within 1 week.

This tertiary centre re-examined the reasons for its delayed discharges and used this information to support the introduction of a deliberate policy to complete training for HPN in the community wherever possible and to establish a new contractual arrangement for HPN, whereby there are parallel contracts between the Hospital Trust and the Primary Care Trust (PCT), and between the Hospital Trust and the homecare provider, replacing the former model in which the hospital negotiated a contract between the PCT and homecare provider, but had no contract itself specific to the HPN.

During the financial year 2006-7 the mean and median intervals from decision to institute HPN and the day of discharge were 101 and $76 \mathrm{~d}$ respectively, the mean reflecting two very delayed discharges of 108 and $210 \mathrm{~d}$. These delays reflected difficulty in obtaining financial agreement, the patients having been medically ready for discharge at days 21 and 63 repectively. In both cases hospital-acquired infections then occurred.

A new contract was offered to European tender and was awarded to the most competitive bidder able to provide the comprehensive national coverage required for a period of 2 years rather than tender on an individual patient basis. This contract has operated in outline since April 2007. Initial responses from PCT were questioning, but, with a single exception, have led to rapid agreement being reached. During the year 2007-8 the mean and median intervals to discharge were 48 and $39 \mathrm{~d}$ respectively (significantly different from 2006-7; $P<0.02$ ). The two outliers (at 82 and $114 \mathrm{~d}$ ) reflected complications of unstable septic Crohn's disease and the patient's initially homeless status and wish to relocate to a different part of the country respectively, rather than procedural or financial delays. Domiciliary nursing to complete HPN training was successfully employed on a temporary basis for three patients who were therefore able to return home sooner.

Unplanned re-admission rates have not changed and patients, hospital staff and the homecare provider all appear to be comfortable with the new arrangements. Quicker high-quality discharge is achieved, without evidence of any new complications and with a more robust arrangement for clinical governance in relation to the continuing care of the patient at home. Such an approach is recommended to all centres joining the home parenteral nutrition and intestinal failure network (HIFNET) partnership. 